EPJ Web of Conferences 70, 00038 (2014)

DOI: 10.1051/epjconf/ 20147000038

(C) Owned by the authors, published by EDP Sciences, 2014

\title{
Can a Future Choice Affect a Past Measurement's Outcome?
}

\author{
Y. Aharonov ${ }^{1}$, E.Cohen ${ }^{1}$, D. Grossman ${ }^{2}$, A.C.Elizutr ${ }^{3}$ \\ ${ }^{1}$ School of Physics and Astronomy, Tel-Aviv University, Tel-Aviv 69978, Israel Conference \\ ${ }^{2}$ Racah Institute of Physics, Hebrew University of Jerusalem, Givat Ram, Jerusalem 91904, Israel \\ ${ }^{3}$ Iyar, The Israeli Institute for Advanced Research, Rehovot, Israel.
}

\begin{abstract}
An EPR experiment is studied where each particle undergoes a few weak measurements along some pre-set spin orientations, whose outcomes are individually recorded. Then the particle undergoes a strong measurement along a spin orientation freely chosen at the last moment. Bell-inequality violation is expected between the two final strong measurements within each EPR pair. At the same time, agreement is expected between these measurements and the earlier weak ones within the pair. A contradiction thereby ensues: i) Bell's theorem forbids spin values to exist prior to the choice of the spin-orientation to be measured; ii) A weak measurement cannot determine the outcome of a successive strong one; and iii) Indeed no disentanglement is inflicted by the weak measurements; yet iv) The weak measurements' outcomes agree with those of the strong ones. The most reasonable resolution seems to be that of the Two-StateVector Formalism, namely, that the experimenter's choice has been encrypted within the weak measurement's outcomes, even before the experimenter themselves knows what their choice will be. Causal loops are avoided by this anticipation remaining encrypted until the final outcomes enable to decipher it.
\end{abstract}

\section{Introduction}

Bell's theorem [1] has dealt the final blow on all attempts to explain the EPR correlations [2] by invoking previously existing local hidden variables. While the EPR spin outcomes depend on the particular combination of spin-orientations chosen for each pair of measurements, Bell proved that the correlations between them are cosine-like and nonlinear Eq. (1) hence these combinations cannot all co-exist in advance. Consequently, nonlocal effects between the two particles have been commonly accepted as the only remaining explanation.

It is possible, however, to explain the results without appeal to nonlocality, by allowing hidden variables to operate according to the Two-State Vector Formalism (TSVF). The hidden variable would then be the future state-vector, affecting weak measurements at present. Then, what appears to be nonlocal in space turns out to be perfectly local in spacetime.

Following is a proof for this account, illustrated in Figure 1. As this proof is bound to elicit attempts to find loopholes within it, we describe it elsewhere in greater detail and with several control experiments [3].

This is an Open Access article distributed under the terms of the Creative Commons Attribution License 2.0, which permits unrestricted use, distribution, and reproduction in any medium, provided the original work is properly cited. 
This paper's outline is as follows. Sec. 2 introduces the foundations of TSVF and 3 introduces weak measurement. 4 describes a combination of strong and weak measurements on a single particle illustrating a prediction of TSVF. In 5 we proceed to the EPR-Bell version of this experiment. Secs.6-8 discuss and summarize the predicted outcomes' bearings.

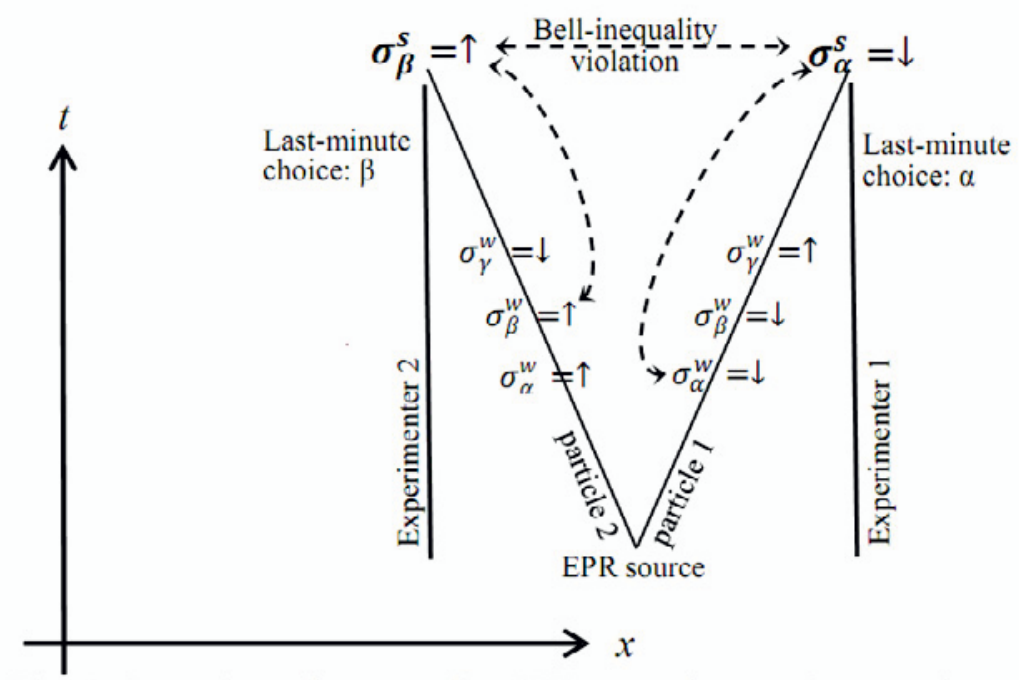

Fig. 1. Spacetime diagram of an EPR experiment where each particle undergoes three pre-set weak measurements and one freely-chosen strong one. The weak measurements turn out to give early records of the strong measurements' results which, by Bell's proof, could not have existed prior to the strong measurements'

\section{The TSVF Formulation of a Particle's State between Two Noncommuting Spin Measurements}

We begin with the TSVF account for a single particle. Consider a large ensemble of $N$ particles, each undergoing two consecutive strong measurements, along the co-planar spin orientations $\alpha$ and $\beta$. The correlation between their outcomes depends on their relative angle $\theta_{\alpha \beta}$ :

$$
<\sigma_{\alpha} \sigma_{\beta}>=\cos \theta_{\alpha \beta}
$$

Also, by the uncertainty relations between spin operators, these two measurements disturb each other's outcomes: If, e.g., the $\alpha$ measurement is repeated after the $\beta$, with $\beta$ being orthogonal to $\alpha$, then $\alpha$ may as well give the opposite value. ABL [4] argued that, at any time between the two measurements, the particle's state is equally determined by both of them. The probability for measuring the eigenvalue $c_{j}$ of the observable $c$, given the initial and final states $\left|\Psi\left(t^{\prime}\right)\right\rangle$ and,$\left\langle\Phi\left(t^{\prime \prime}\right)\right|$ respectively, is described by the symmetric formula:

$$
P\left(c_{j}\right)=\frac{\left|\left\langle\Phi(t) \mid c_{j}\right\rangle\left\langle c_{j} \mid \Psi(t)\right\rangle\right|^{2}}{\sum_{i}\left|\left\langle\Phi(t) \mid c_{i}\right\rangle\left\langle c_{i} \mid \Psi(t)\right\rangle\right|^{2}}
$$


The probability thus seems to have a definite value which agrees with both outcomes, due to two state-vectors [5], one evolving from the past,

$$
|\psi(t)\rangle=\exp \left(\int_{t^{\prime}}^{t}-i H / \hbar d t\right)\left|\psi\left(t^{\prime}\right)\right\rangle, \quad t>t^{\prime}
$$

and the other from the future:

$$
\langle\Phi(t)|=\left\langle\Phi\left(t^{\prime \prime}\right)\right| \exp \left(\int_{t^{\prime \prime}}^{t} i H / \hbar d t\right), \quad t<t^{\prime \prime}
$$

creating the two-vector

$$
\left\langle\Phi\left(t^{\prime \prime}\right)|| \psi\left(t^{\prime}\right)\right\rangle
$$

\section{Weak measurements}

It is for the detection of such delicate intermediate states that weak measurement [5] has been conceived. Weak measurement couples each spin to a device whose pointer moves $\lambda / \sqrt{N}$ or - $\lambda / \sqrt{N}$ units upon measuring, respectively, $\uparrow$ or $\downarrow$, when $N$ is the size of the ensemble and $\lambda$ is a constant. Let the pointer value have a Gaussian noise with 0 expectation and $\delta>\lambda / \sqrt{N}$ standard deviation. When measuring a single spin, we get most of the results within a wide range $\lambda / \sqrt{N} \pm \delta$, but when summing up the $N / 2$ results, we find most of them within a much narrower range $\lambda \sqrt{N} / 2 \pm \delta \sqrt{N} / \sqrt{2}$ thereby agreeing with the strong result when $\lambda \gg \delta$.

It is on large ensembles of particles that weak measurement's efficiency becomes evident. Let $N$ particles undergo an interaction Hamiltonian of the form

$$
H \operatorname{int}(t)=\frac{\lambda}{\sqrt{N}} g(t) A_{s} P_{d}
$$

Where $A_{s}$ denotes the measured observable and $P_{d}$ is canonically conjugated momentum to $Q_{d}$, representing the measuring device's pointer position. The coupling $\mathrm{g}(\mathrm{t})$ is nonzero only for the time interval $0 \leq t \leq T$ and normalized according to

$$
\int_{0}^{T} g(t) d t=1
$$

The measurement's weakness (and consequently strength) is due to the small factor ${ }^{2} \lambda / \sqrt{N}$, inversely proportional to the ensemble's size, where $\lambda$ is a constant.

\footnotetext{
${ }^{\mathrm{a}} \mathrm{Weakness}$ of $1 / \mathrm{N}$ is sufficient in this case where one measuring apparatus is used, but for the cases considered in the next sections we chose $1 / \sqrt{N}$ interaction strength. See also [4] and [5].
} 
When the $N$ particles have different states, e.g., spins, the weak measurement correctly gives their average. But when they all share the same $\uparrow$ or $\downarrow$ spin value along the same orientation, weak measurement indicates that its outcome gives the entire ensemble's state. As pointed out in [4]:

$$
\langle\bar{A}\rangle_{w}=\frac{1}{N} \sum_{n=1}^{N}\left\langle A^{(n)}\right\rangle_{w}=\langle\psi|A| \psi\rangle
$$

i.e., $\vec{A}$ s weak value approaches the expectation value of $A$ operating on $|\psi\rangle$. The weak measurement's precision thus guarantees its agreement with the strong measurement.

\section{Combining Strong and Weak Measurements}

We are now in a position to give a thought-experimental demonstration of the claim made in Sec. 2: A particle's state between two strong measurements carries both the past and future outcomes. Consider again an ensemble of $N$ particles. Then,

\subsection{Procedure}

a) On morning Bob strongly measures all particles' spins along the $\alpha$-orientation. He measures them one by one and assigns them serial numbers, such that each particle remains individually distinct throughout all measurements, as in a "single shot" experiment.

b) On noon Alice weakly measures all particles' spins along the $\alpha$ and $\beta$ orientations plus a third coplanar orientation $\gamma$. Her measurements are similarly individual, each particle measured in its turn, and the measuring device calibrated before the next measurement. For reasons explained below, she repeats this series 3 times, total 9 weak measurements per each particle. All lists of outcomes ${ }^{3}$ are then publically recorded, e.g., engraved on stone (see Figure 2), along 9 rows. Summing up her $\alpha$-measurements (whether $\alpha^{(1)}, \alpha^{(2)}, \alpha^{(3)}$ separately or all $3 N$ together), she finds the spin distribution $50 \% \uparrow-50 \% \downarrow$. Similarly for $\beta$ and $\gamma$.

c) On evening Bob, oblivious of Alice's noon outcomes, again strongly measures all $\mathrm{N}$ particles, this time along the $\beta$ orientation. He then draws a binary line $\neg$ along his row of outcomes such that all $\uparrow$ outcomes are above the line and all $\downarrow$ 's below it.

d) Bob then gives Alice the two lists of his morning and evening outcomes. The lists are coded, such that $\mathrm{x} / \mathrm{y} / \mathrm{z}$ stand for $\alpha / \beta / \gamma$ and "above line"/"below line" for $\uparrow / \downarrow$.

e) Based on Bob's lists, Alice slices her data, recorded since morning, according to Bob's divisions. In terms of Figure 2, she merely shifts each of the $\neg$ lines from Bob's lists to each one of her 9 rows of outcomes carved on stone. Each of the $N$ sequences is thereby split into two N/2 sub-rows, one above and the other below the binary line. She then re-

\footnotetext{
${ }^{3}$ Although each measurement's result can be any real number, for simplicity we describe Alice's results as binary - "up" for positive numbers and "down" for negative numbers.
} 
sums each half separately. Each row is thus sliced twice, first according to Bob's morning strong measurements' list and then according to his evening list.

\subsection{Predictions}

Upon Alice's re-summing up her each of sliced lists, QM obliges the following:

a) Out of the 9 sliced rows of the weak measurements' outcomes, 3 immediately stand out with maximal correlation with Bob's above/belowx list, indicating that $\mathrm{x}=\alpha$, above $=\uparrow$ below $=\downarrow$. Similarly for Bob's evening above/belowy list: 3 other rows show that $y=\beta$, above $=\uparrow$ below $=\downarrow$. In short, all weak measurements agree with the strong ones, whether performed before or after them, to the extent that enables Alice to exactly point out which particle was subjected to which spin measurement by Bob, and what was the outcome.

b) Hence, all same-spin weak measurements confirm one another.

c) Even the third spin orientation weakly measured by Alice, $\gamma$, is correlated with $\alpha$ and $\beta$ according to the same probabilistic relations of Eq. (1).

d) Even in case Bob's measurement is along an orientation other than $\alpha, \beta$, or $\gamma$, Alice's data can precisely reveal this orientations, as well as all the individual spin values, by employing the (1) relations.

a. Weak values (presented for convenience as

binary), engraved in stone on noon.

$n \uparrow=n \downarrow=N / 2$
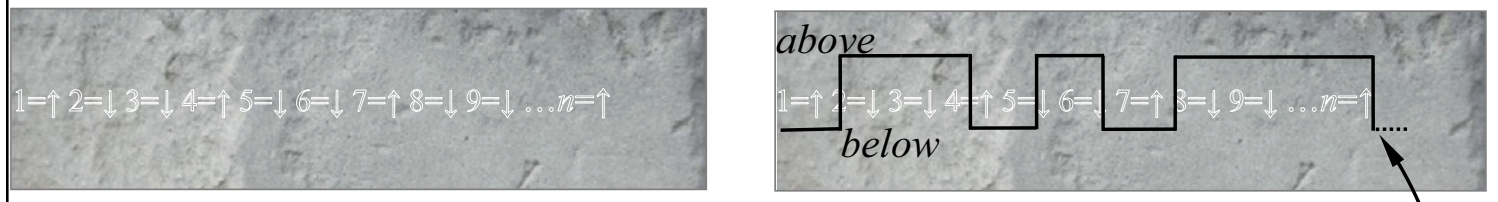

c. The evening outcomes' slicing is applied to the noon outcomes.

b. Strong values, recorded on evening and $n \uparrow_{\text {above }}>\mathrm{n} \downarrow_{\text {above }}, n \uparrow_{\text {below }}<\mathrm{n} \downarrow_{\text {below }}$ sliced into above/below.

above
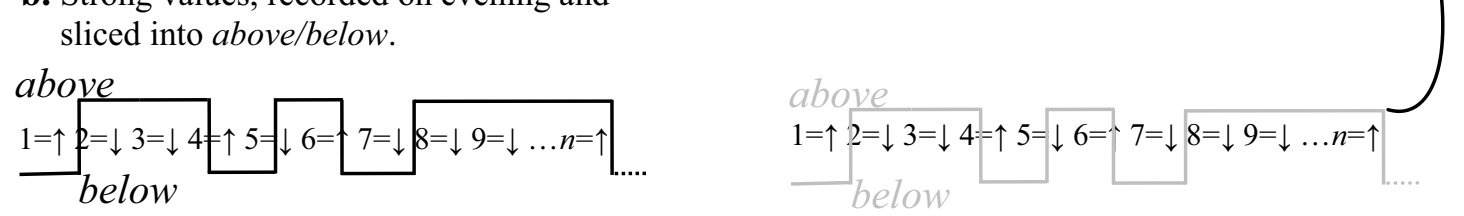

Fig. 2.The time-order of weak followed by strong measurements, and the slicing method indicating the latter's outcomes encryption within the former. For simplicity, Alice's weak results are presented as binary numbers (up or down). 
These predictions are unique in two respects. The weak measurements results precisely repeat themselves despite the fact that, for each pair of same-spin weak measurements, two noncommuting measurements were made between them. For example, the spin along the $\alpha$-orientation remains the same upon the next weak spin $\alpha$ measurement despite the intermediate $\beta$ and $\gamma$ spin measurements.

Even more striking is the fact that all weak measurements equally agree with the past and future strong measurements. While it is not surprising that the noon weak measurements confirm the morning strong outcomes, it is certainly odd that they anticipate the evening ones.

This fully accords with the TSVF. Mainstream physics, however, would prefer a simpler explanation. Perhaps, e.g., the weak measurements introduce some subtle kind of $\beta$ collapse, which the later strong $\beta$ measurements' outcomes simply reaffirms, despite the intermediate $\alpha$ and $\beta$ weak measurements.

We have carefully considered this possibility elsewhere [6] and proved its inadequacy. Moreover, our next experiment would be much harder to account for along these one-vector lines. Other routes for proving this point are discussed in [9][10].

\section{Combining Strong and Weak Measurements In the EPR-Bell Experiment}

We can now demonstrate the weak outcomes' anticipation of a future human free choice. Consider an EPR-Bell experiment $[1,2]$ on an ensemble of $N$ particle pairs.

\subsection{Procedure}

a) On morning, Alice carries out 9 weak measurements on each particle within each EPR pair, 3 measurements along each orientation, $\alpha, \beta$ and $\gamma$ (with the coupling strength appropriately weakened). Every result is recorded, alongside with the pair's serial number among the $N$, the particle's identity (Right/Left) within the pair, and the weak measurement's number among the 9 (Figure 3). The entire list is then engraved on stone (Figure 2) along 9 rows.

b) On evening, Bob, oblivious of Alice's data, performs one strong spin measurement on each particle. For simplicity, he chooses only one spin-orientation for all right-hand particles and one for all left-hand ones. With sufficiently large $N$, he can choose a pair of measurements anew for each pair of particles. The crucial fact is this: The spin orientations are chosen at the last moment by Bob's free choice.

c) Bob sends Alice a list of his outcomes in which the spin orientations and values are coded: $x / y / z$ for $\alpha / \beta / \gamma$ and above/below for $\uparrow / \downarrow$.

d) Based on Bob's lists, Alice slices her data, carved on stone since morning, according to Bob's divisions, again shifting the binary line 7 from each of Bob's lists to her rows, as in $\mathrm{Sec} 4$. 


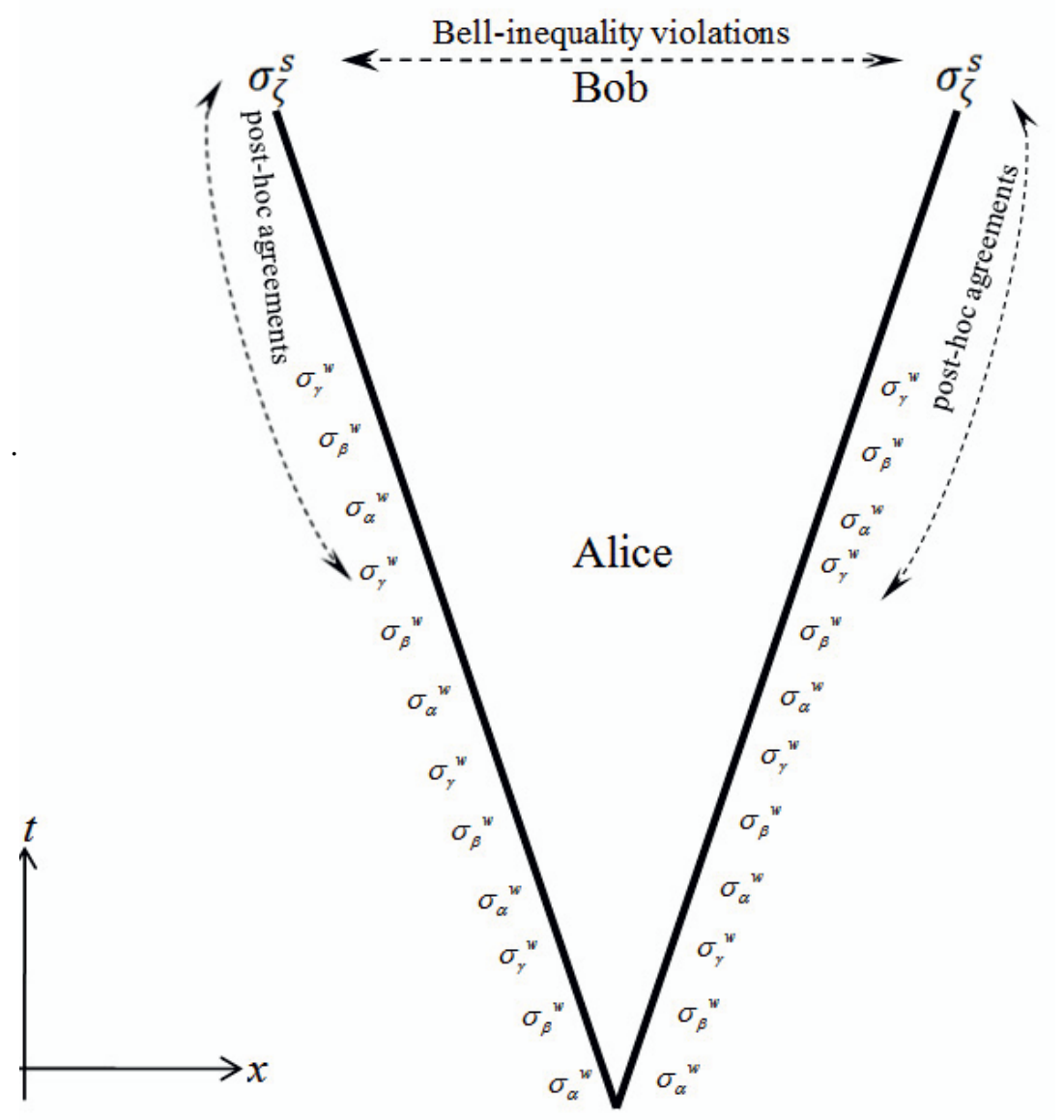

Fig. 3. An EPR setting with several weak measurements followed by strong ones.

\subsection{Procedure}

Calculating the new separate averages of each sub-ensemble, QM obliges the following (a statement about a weak measurement refers to its overall outcome):

a) Bob's pairs of strong measurements' outcomes exhibit the familiar Bell-inequality violations [1], indicating that the particles were superposed prior to his measurements.

b) Alice's weak outcomes strictly agree with those of the strong measurements, exhibiting similar Bell-correlations;

c) with the following addition: For each particle, the strong measurement carried out on it determine the other particle's spin as if they occurred in the other particle's past, with the $\uparrow / \downarrow$ sign inverted, regardless of the measurements' actual timing. 


\section{Will One Vector Do?}

Naturally, more conservative interpretations ought to be considered before concluding that measurements' results anticipate a future event. By normal causality, it must be Alice's results which affected Bob's, rather than vice versa. It might be, for example, some subtle bias induced by her weak measurements later to affect his strong ones.

Such a past-to-future effect can be straightforwardly ruled out by posing the following question: How robust is the alleged bias introduced by the weak measurements? If it is robust enough to oblige the strong measurements, then it is equivalent to full collapse, namely local hidden variables, already ruled out by Bell's inequality. However, weakly measured particles remain nearly fully entangled, thereby excluding such bias. But then, even the weakest bias, as long as it is expected to show up over a sufficiently large $N$, is ruled out of the same grounds. The "weak bias" alternative is ruled out also by the robust correlations predicted between all same-spin measurements, whether weak or strong.

Another way to disprove the one-vector account is by the following question: Can Alice predict Bob's outcomes on the basis of her own data? To do that, she must feed all her rows of outcomes into a computer that searches for a possible series of spin-orientation choices plus measurement outcomes, such that, when she slices her rows accordingly, she will get the complex pattern of correlations described above.

The number of such possible sequences that she gets from her computation is $\left(\begin{array}{c}N \\ N / 2\end{array}\right) \propto \frac{2^{N}}{\sqrt{N}}$.

Each such sequence enables her to slice each of her rows into two $N / 2$ halves and get the above correlations between her weak measurements and the predicted strong measurements. Notice that, according to Sec. 2, the results' distribution is a Gaussian with $\lambda \sqrt{N} / 2$ expectation and $\delta \sqrt{N} / 2$ standard deviation, so a $\delta$ shift in one of the results, or even $\sqrt{N}$ of them, is very probable. Hence, even if Alice computes all Bob's possible future choices, she still cannot tell which choice he will take, because there are many similar subsets giving roughly the same value. Also, as Aharonov et al. pointed out in [3], when Alice finds a subset with a significant deviation from the expected 50$50 \%$ distribution, its origin is much more likely, upon a real measurement by Bob, to turn out to be a measurement error than a genuine physical value. Obviously, then, present data is insufficient to predict the future choice.

\section{What Kind Of Causality?}

Regardless, therefore, of the above result's oddity from mainstream QM's view, they fully accord with the TSVF account of backward-propagating causal effects in the quantum realm.

Recall first Bell's proof: For an entangled pair, no set of spin values can exist beforehand so as to give the predicted correlations for all possible choices of spin orientations to be measured.

Applied to our setting, this prohibition seems to allow only the following account: 
a) On morning, several weak spin measurements were performed on $N$ particles, resulting in even $\uparrow / \downarrow$ distributions. These outcomes were recorded, thereby becoming definite and irreversible.

b) Then on evening, all the particles underwent strong measurements, on spin orientations chosen randomly, hence unknown beforehand, even to the experimenter himself.

c) All these evening measurements exhibited Bell inequality violations within each pair.

d) Next, all the morning lists were sliced in accordance with the evening outcomes.

e) Unequivocal correlations emerged between all the morning and evening outcomes.

f) By Bell's theorem, the particle pairs could not have been correlated on morning for whatever possible spin-orientations that might be chosen to be measured on evening.

g) Neither could the strong measurements' outcomes have been determined by the weak measurements, for, in that case, the particles would be disentangled already on morning, failing to violate Bell's locality on evening.

h) Ergo, the weak measurements' agreement with the strong measurements could have been obtained only by the former giving early records of the spin orientation to be chosen for the latter. This result indicates the existence of a hidden variable of a very subtle type, namely the future state-vector.

The addition of weak measurements to the EPR experiment thus gives, for the first time, an empirical evidence that the measurement of each particle's within the EPR pair is equivalent to the other particle's pre-selection.

\section{Summary}

The addition Our proof rests on two well-established findings: $i$ ) Bell's nonlocality theorem and ii) The causal asymmetry between weak and strong measurements.

The EPR-Bell experiment proves that one particle's spin outcome depends on the choice of the spin-orientation to be measured on the other particle, and its outcome thereof. Relativistic locality is not necessarily violated in this experiment, as it allows either Alice's choices to affect Bob's, or vice versa.

This reciprocity, however, does not hold for a combination of measurements of which one is weak and the other strong. The latter affects the former, never vice versa. Therefore, when a weak measurement precedes a strong one, the only possible direction for the causal effect seems to be from future to past. 
We stress again that attempt to dismiss the weak measurement's peculiar outcomes by invoking some subtle collapse due to the weak measurement, or any other form of contaminating the initial superposed states, have been thoroughly considered and ruled out [1] [6] [7].

Also, while earlier predictions derived from the TSVF were sometimes dismissed as counterfactuals, there is nothing counterfactual in the experiments proposed in this paper. Our predictions refer to actual measurements whose outcomes are objectively recorded. Moreover, our experiment turns even the counterfactual part of the EPR experiment into an actual physical result: Prediction (c) in subsection 4.2 refers to a spin-orientation not eventually chosen for strong measurements, thereby being a mere "if" in the ordinary EPR experiment. In our setting, even this unperformed choice yields actual results through the weak measurements.

Finally, this experiment sheds a new light on the age-old question of free will. Apparently, a measurement's anticipation of a human choice made much later renders the choice fully deterministic, bound by earlier causes. One profound result, however, shows that this is not the case. The choice anticipated by the weak outcomes can become known only after that choice is actually made. This inaccessibility, which prevents causal paradoxes like "killing one's grandfather," secures human choice full freedom from both past and future constraints. A rigorous proof for this compatibility between TSVF and free choice is given elsewhere in detail [8].

\section{Acknowledgements}

It is a pleasure to thank Shay Ben-Moshe, Paz Beniamini, Shahar Dolev, Yuval Gefen, Einav Friedman, Ruth Kastner Marius Usher for helpful comments and discussions. This work has been supported in part by the Israel Science Foundation Grant No. 1125/10.

\section{References}

1. J. Bell, Physics 1 (3), 195-200 (1964).

2. A. Einstein, B. Podolsky, N. Rosen, Phys. Rev. 47, 777-780 (1935).

3. Y. Aharonov, E. Cohen, A. C. Elitzur, PRL, (to be published).

4. Y. Aharonov, P.G. Bergman, J.L. Lebowitz, Phys. Rev. 134 (1964).

5. Y. Aharonov, L. Vaidman, , Phys. Rev. A 41, 11-20 (1990).

6. Y. Aharonov, E. Cohen, A. C. Elitzur, Phys. Rev. A (to be published).

7. Y. Aharonov, E. Cohen, A. C. Elitzur, Phys. Rev. A (to be published).

8. Y. Aharonov, E. Cohen, A. C. Elitzur, PRL, (to be published).

9. Y. Aharonov, E. Cohen, S. Ben-Moshe, International Conference on New Frontiers in Physics (to be published).

10. Y. Aharonov, E. Cohen, A. C. Elitzur, PRL, (to be published). 